\title{
Circulating microRNA alternations in primary hyperuricemia and gout
}

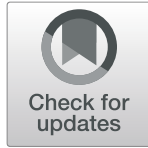

Jana Bohatá ${ }^{1,2}$, Veronika Horváthová ${ }^{1,3}$, Markéta Pavlíková ${ }^{4}$ and Blanka Stibůrková1, ${ }^{*}$

\begin{abstract}
Objectives: MicroRNAs (miRNAs) are short single-stranded RNAs that play a role in the post-transcriptional regulation of gene expression. Their deregulation can be associated with various diseases, such as cancer, neurodegenerative, and immune-related diseases. The aim of our study was to compare miRNA levels in plasma that could potentially influence the progression of hyperuricemia to gout, since the mechanism of progression is still unclear.

Methods: Total RNA, including miRNA, was isolated from the plasma of 45 patients with asymptomatic hyperuricemia, 131 patients with primary gout (including 16 patients having a gout attack), and 130 normouricemic controls. The expression of 18 selected miRNAs (cel-miR-39 and cel-miR-54 as spike-in controls, hsa-miR-16-5p and hsa-miR-25-3p as endogenous controls, hsa-miR-17-5p, hsa-miR-18a-5p, hsa-miR-30a-3p, hsa-miR-30c-5p, hsa-miR126-3p, hsa-miR-133a-3p, hsa-miR-142-3p, hsa-miR-143-3p, hsa-miR-146a-5p, hsa-miR-155-5p, hsa-miR-222-3p, hsamiR-223-3p, hsa-miR-488-3p and hsa-miR-920) was measured using qPCR.

Results: We found that hsa-miR-17-5p, hsa-miR-18a-5p, hsa-miR-30c-5p, hsa-miR-142-3p, and hsa-miR-223-3p were significantly upregulated $(p<0.001)$ in the plasma of hyperuricemia and gout patients compared to normouricemic individuals. As part of the follow-up of our previous study, we found a negative correlation between hsa-miR-17-5p, hsa-miR-30c-5p, hsa-miR-126-3p, hsa-miR-142-3p, and hsa-miR-223-3p with plasma levels of chemokine MCP-1. Additionally, we found a positive correlation between CRP and plasma levels of hsa-miR-17-5p, hsa-miR-18a-5p, hsamiR-30c-5p, hsa-miR-126-3p, hsa-miR-142-3p, hsa-miR-146a-5p, hsa-miR-155-5p, hsa-miR-222-3p, and hsa-miR-2233p. Five of those miRNAs (hsa-miR-126-3p, hsa-miR-142-3p, hsa-miR-146a-5p, hsa-miR-155-5p, and hsa-miR-222-3p) also had a positive correlation with serum creatinine and therefore a negative correlation with eGFR.

Conclusion: Five miRNAs were significantly upregulated in the plasma of patients with hyperuricemia and gout (and those during a gout attack) compared to normouricemic controls. We also found a correlation between the plasma levels of several miRNA and plasma levels of MCP-1, CRP, serum creatinine, and eGFR.
\end{abstract}

Keywords: miRNA, Uric acid, Hyperuricemia, Gout, Acute gouty arthritis

\footnotetext{
*Correspondence: stiburkova@revma.cz

'Institute of Rheumatology, Prague, Czech Republic

${ }^{5}$ Department of Pediatrics and Inherited Metabolic Disorders, First Faculty of

Medicine, Charles University and General University Hospital, Prague, Czech Republic

Full list of author information is available at the end of the article
}

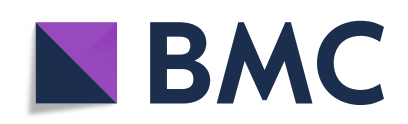

(- The Author(s). 2021 Open Access This article is licensed under a Creative Commons Attribution 4.0 International License, which permits use, sharing, adaptation, distribution and reproduction in any medium or format, as long as you give appropriate credit to the original author(s) and the source, provide a link to the Creative Commons licence, and indicate if changes were made. The images or other third party material in this article are included in the article's Creative Commons licence, unless indicated otherwise in a credit line to the material. If material is not included in the article's Creative Commons licence and your intended use is not permitted by statutory regulation or exceeds the permitted use, you will need to obtain permission directly from the copyright holder. To view a copy of this licence, visit http://creativecommons.org/licenses/by/4.0/. The Creative Commons Public Domain Dedication waiver (http://creativecommons.org/publicdomain/zero/1.0/) applies to the data made available in this article, unless otherwise stated in a credit line to the data. 


\section{Introduction}

Uric acid is the end product of purine metabolism in the human body. Two-thirds of which is excreted by the kidneys and one-third is excreted by the gastrointestinal tract. Hyperuricemia is caused by excessive production of uric acid (10\% of cases) and/or reduced excretion (the majority of cases); about $10 \%$ of hyperuricemia cases escalate to clinically definable gout. There are risk factors for hyperuricemia and gout, e.g., lifestyle, metabolic syndrome, age, sex, and genetic predispositions. Major genes affecting uric acid excretion and reabsorption are $A B C G 2$, respectively $S L C 2 A 9$ and $S L C 22 A 12$, which encode membrane transporters ABCG2, GLUT9, and URAT1. Variants in the $S L C 22 A 12$ gene can cause renal hypouricemia type 1 (OMIM 220150); similarly, variants in the $S L C 2 A 9$ gene can cause renal hypouricemia type 2 (OMIM 612076). SLC2A9 is sometimes present in association with hyperuricemia and gout, but this has not been confirmed by functional analysis [1]. The most relevant gene that plays a role in hyperuricemia and gout is $A B C G 2$. For example, variants Q126X (rs72552713) and Q141K (rs2231142) in the ABCG2 gene can cause severe dysfunction in this transporter and account for $90 \%$ of early-onset gout patients [2]. In our previous studies, we published on the influence of nonsynonymous allelic variants, including functional characterizations, relative to the increased risk of gout progression [3, 4].

Gout is a common inflammatory arthritis. The condition is caused by an accumulation of monosodium urate (MSU) crystals in tissues, which can sometimes lead to gout flare-ups (flares). MSU crystals initiate a macrophage reaction, which activates NLRP3 inflammasomes leading to a release of interleukine-1 $\beta$ (IL-1 $1 \beta$ ). IL- $1 \beta$ is a crucial cytokine in gout flares. However, patients with hyperuricemia can also have MSU crystal deposits [5]. An acute gout flare is conditioned not only on activation of NLRP3 inflammasomes but also on upregulation of IL1B transcription. IL-1 $\beta$ and other interleukins (IL-6, IL-8) attract neutrophils to the site of inflammation resulting in an acute gout flare [6].

The mechanism of hyperuricemia to gout progression is still not fully understood. Gout has four stages of development: asymptomatic hyperuricemia, acute gouty arthritis, intercritical gout, and chronic tophaceous gout. There are probably many variables influencing the progress, and they can vary for each individual.

This study seeks to determine if miRNAs are variable factors that can stimulate the development of gout from asymptomatic hyperuricemia. According to the first GWAS study addressing aggravation of asymptomatic hyperuricemia to gout, there were three genetic loci associated with hyperuricemia to gout development. One of them is rs9952962 near miR-302f [7]. Finding indicators of hyperuricemia to gout development is an important step in preventive gout therapy.

MicroRNAs (also miRNAs) are short single-stranded non-coding RNAs that play a role in the regulation of gene expression. These molecules were first described in 1993 in Caenorhabditis elegans [8]. MiRNAs are involved in the normal function of cells; however, their dysregulation is associated with various diseases. MiRNAs are present in cells as intracellular miRNAs, or they are released from cells into the extracellular environment (often within extracellular vesicles) and are called extracellular, circulating, or cell-free miRNAs, where they play a role in intercellular communication [9]. Cell-free miRNAs are potentially suitable as non-invasive or minimally invasive biomarkers [10]. There are many studies about the role of miRNA in cancer, cardiovascular diseases, immune-related diseases, and others, but only a few studies have dealt with miRNA in hyperuricemia and gout. More than $60 \%$ of human protein-coding genes are targets for various miRNA [11]. There are different ways miRNAs can influence serum uric acid and the mechanism of gout pathophysiology. MiRNAs can regulate the expression of urate transporters genes, for example, miR-34a targets mRNA of the SLC22A12 gene and inhibits expression of URAT1 in hyperuricemic animal models [12]. Further, miRNAs can influence the expression of essential enzymes such as xanthine oxidase, which can be regulated by miR-448 [13]. Another way of miRNA interference is regulating the expression of genes involved in gout immune response, e.g., miR-223 can suppress NLRP3 expression and reduce inflammasome activity [14]. We aimed to detect and confirm circulating miRNAs that are expressed differently in hyperuricemia and/or gout. Further, we wanted to apply data from our miRNA analysis to our previous research addressing plasma cytokine levels in the same cohort of patients. The association between miRNA levels and biochemical/clinical parameters was also studied.

\section{Methods}

\section{Sample collection/cohort}

The study cohort comprised 115 patients (147 samples; 83 patients with one measurement and 32 patients with two measurements) with primary gout (118 males, 13 females; median age 53 years), 16 patients having a gout attack (24 samples; 10 patients with one measurement, five patients with two measurements, and one patient with four measurements), 45 patients (54 samples; 36 patients with one measurement and nine patients with two measurements) with asymptomatic hyperuricemia (33 males, 12 
females; median age 48 years), and 130 normouricemic controls (53 males, 77 females; median age 41 years). All plasma samples were stored at $-80^{\circ} \mathrm{C}$ in the biobank of the Institute of Rheumatology, Prague, the Czech Republic. Gout patients met the 1977 American Rheumatism Association preliminary classification criteria [15]. Primary hyperuricemic patients were classified as having serum uric acid (SUA) > $420 \mu \mathrm{mol} / \mathrm{L}$ for men and SUA $>360 \mu \mathrm{mol} / \mathrm{L}$ for women. Patients were compared to 130 normouricemic individuals from the general population, with no history of primary hyperuricemia, gout, or autoimmune disease. We used the same group of patients used in a previous study addressing plasma cytokines levels in these patients [16]. Written informed consent was obtained from each subject before enrollment in the study. All tests were performed in accordance with standards set by the institutional ethics committees, which was approved 30 June 2015, the project no. 6181/2015. All the procedures were performed in accordance with the Declaration of Helsinki. All demographic, biochemical, genetic, and presence and type of medical treatment data of patients and normouricemic individuals are presented in Table 1.

\section{miRNA analysis}

We performed a screening of expressed miRNAs in 12 samples -3 representatives of each studied group (normouricemic controls, patients with hyperuricemia, patients with gout, patients during a gout attack). For screening, TLDA cards were used-TaqMan ${ }^{\text {Thx }}$ Array Human MicroRNA A+B Cards Set v3.0 (ThermoFisher). We got results for 754 miRNAs, and subsequently, we were able to choose miRNAs that showed potentially different expressions between the studied cohorts but not within them (hsa-miR-17-5p, hsa-miR-18a-5p, hsa-miR30c-5p, hsa-miR-133a-3p, hsa-miR-142-3p, hsa-miR143-3p, and hsa-miR-222-3p). Using TaqMan ${ }^{\text {Tu }} \mathrm{Ad}-$ vanced miRNA Human Endogenous Controls Card (ThermoFisher) and NormFinder software, we selected two stable endogenous control miRNAs (hsa-miR-16-5p and hsa-miR-25-3p). We also did a review of published studies and found potential target miRNAs. According

Table 1 Main demographic, biochemical, and genetic characteristics of patients

\begin{tabular}{|c|c|c|c|c|c|c|c|}
\hline & \multicolumn{2}{|c|}{ Normouricemic subjects $(n=130)$} & \multicolumn{2}{|c|}{ Hyperuricemic patients ( $n=45$ ) } & \multicolumn{2}{|c|}{ Gout patients $(n=131)$} & \multirow[t]{2}{*}{ Fisher test $p$-value } \\
\hline & $n$ & $\%$ & $\mathrm{n}$ & $\%$ & $\mathrm{n}$ & $\%$ & \\
\hline Sex M/F & $53 / 77$ & $40.8 / 59.2$ & $33 / 12$ & $73.3 / 26.7$ & $118 / 13$ & $90.1 / 9.9$ & $<0.0001$ \\
\hline Familial occurrence & & & 17 & 41.5 & 50 & 38.5 & 0.8546 \\
\hline No treatment & 130 & 100 & 21 & 46.7 & 18 & 13.7 & $<0.0001$ \\
\hline Allopurinol & No treatment & & 24 & 53.3 & 98 & 74.8 & $<0.0001$ \\
\hline Febuxostat & & & 0 & 0 & 15 & 11.5 & $<0.0001$ \\
\hline p.Q141K, GG & 102 & 87.2 & 25 & 58.1 & 73 & 56.6 & $<0.0001$ \\
\hline GT & 14 & 12.0 & 13 & 30.2 & 49 & 38.0 & $<0.0001$ \\
\hline$\pi$ & 1 & 0.9 & 5 & 11.6 & 7 & 5.4 & $<0.0001$ \\
\hline no genetic data & 13 & 10.0 & 2 & 4.4 & 2 & 1.5 & $<0.0001$ \\
\hline \multirow[t]{2}{*}{ p.Q141K, MAF } & 16 & 6.8 & 23 & 26.7 & 63 & 24.4 & $<0.0001$ \\
\hline & Median (IQR) & Range & Median (IQR) & Range & Median (IQR) & Range & KW test $p$-value \\
\hline Age of onset & Not applicable & & $34(40.5)$ & $6-76$ & $41.0(23.0)$ & $12-84$ & 0.197 \\
\hline Age at the time of taking samples & $41(25.0)$ & $18-76$ & $48.0(49.0)$ & $11-78$ & $53.0(20.5)$ & $14-90$ & $<0.0001$ \\
\hline BMI & $25.3(4.8)$ & $17.9-38.5$ & $28.7(6.1)$ & $17.7-41$ & $28.6(5.4)$ & $20.6-50$ & $<0.0001$ \\
\hline SUA off treatment, $\mu \mathrm{mol} / \mathrm{L}$ & $337.0(118.8)$ & $140-617$ & $450.5(105.0)$ & $253-601$ & $462.0(124.0)$ & $245-683$ & $<0.0001$ \\
\hline SUA on treatment, $\mu \mathrm{mol} / \mathrm{L}$ & Not applicable & & $424.0(143.0)$ & $250-608$ & $378.0(124.0)$ & $167-725$ & 0.2325 \\
\hline FE-UA & Not collected & & $3.9(2.0)$ & $1.8-20$ & $3.6(1.5)$ & $0.8-14.3$ & 0.1716 \\
\hline eGFR-MDRD, mL/min/1.73 m2 & Not collected & & $88.0(35.6)$ & $27.6-165$ & $86.0(21.5)$ & $27.5-151$ & 0.5505 \\
\hline Serum creatinine, $\mu \mathrm{mol} / \mathrm{L}$ & $75.5(21.8)$ & $49-121$ & $79.0(19.0)$ & $47-132$ & $81.0(16.5)$ & $48-189$ & 0.0075 \\
\hline Max CRP & $1.3(1.8)$ & $0.1-17.9$ & $1.9(4.6)$ & $0.2-45.3$ & $4.1(7.2)$ & $0.2-224.4$ & $<0.0001$ \\
\hline
\end{tabular}

Reference range: SUA $120-416 \mu \mathrm{mol} / \mathrm{L}$ for men, $120-360 \mu \mathrm{mol} / \mathrm{L}$ for women; FE-UA $7.3+1.3 \mathrm{for} \mathrm{men}, 10.3 \pm 4.2$ for women; eGFR-MDRD > 90 mL/min/1.73 m² for healthy subjects (levels decline with age); serum creatinine 64-104 $\mu \mathrm{mol} / \mathrm{L}$ for men, $49-90 \mu \mathrm{mol} / \mathrm{L}$ for women; CRP 0-5 mg/L

GG-wild-type variant; GT-heterozygotic; T-homozygotic; MAF minor frequency allele, IQR interquartile range, BMI body mass index, SUA serum uric acid, FE$U A$ fractional excretion of uric acid, eGFR-MDRD estimated glomerular filtration rate calculated using the Modification of Diet in Renal Disease, CRP C-reactive protein, KW test Kruskal-Wallis test 
to those published studies, we chose other miRNAs (hsa-miR-30a-3p, hsa-miR-126-3p, hsa-miR-146a-5p, hsa-miR-155-5p, hsa-miR-223-3p, hsa-miR-488-3p, and hsa-miR-920), and we also confirmed our TLDA selection (Table 2, Fig. 1). The names of all miRNAs are abbreviated in the following text as miR-x.

Cell-free RNA, including miRNA, was isolated from plasma using the miRNeasy Serum/Plasma kit (Qiagen). During isolation, we added the first spike-in control $(0.1 \mathrm{nmol}$ cel-miR-39) to check the isolation efficiency between samples. All plasma samples were prepared using standard protocols and stored at $80^{\circ} \mathrm{C}$ until isolation. Next, we performed reverse transcription using TaqMan $^{\text {Tu }}$ Advanced miRNA cDNA Synthesis Kits (ThermoFisher). The initial step in reverse transcription was the addition of a second spike-in control $(0.1 \mathrm{nmol}$ cel-miR-54), which was used to verify its efficiency. This process contains four steps: (1) 3' poly-A tailing, (2) 5' ligation of an adaptor sequence, (3) reverse transcription to cDNA, and (4) miRNA amplification. The last step of the analysis was qPCR using TaqMan ${ }^{\text {nu }}$ Advanced miRNA Assays specific for each individual target miRNA (ThermoFisher). qPCR was done using a QuantStudio 7 Flex Real-Time PCR System. For data normalization, levels of endogenous miR-25-3p were used.

\section{Statistical analysis}

Continuous variables were summarized as medians with interquartile range (IQR). Demographic and anamnestic variables in normouricemic, hyperuricemic, and gout cohorts were compared using the Kruskal-Wallis test for continuous and Fisher's exact test for categorical variables.

Missing data and discordance between replicates in miRNA data were treated using the method published by de Ronde et al. [30] for duplicates; the efficiency coefficient was set to 1.8. For each miRNA and each individual, the mean quantification cycle $(\mathrm{Cq})$ value was computed as the mean of the two duplicates. Undetectable $\mathrm{Cq}$ values (over 35 ) were replaced by the maximal measured $\mathrm{Cq}+1$ for each respective miRNA. We first considered replacing discordant duplicates (the difference between duplicates larger than 0.5) with multiple imputation MICE (Multivariate Imputation by Chained Equations) according to the patient's age, sex, and diagnosis. However, those imputed values varied even more than the original measurements: e.g., for miR-25, the most discordant replicates differed by 1.1 while some of the imputed values differed by more than three from the mean of the duplicates. We, therefore, opted for keeping the mean of the discordant duplicates for the reference miR-25 (18 duplicates in total, representing $5.1 \%$ of all measurements) and not using discordant replicates for the other targeted miRNA. Sensitivity analysis using original and imputed values for reference miRNA showed no substantial difference in $p$-value estimates. An overview of undetectable, valid, and invalid replicates is given in Supplementary Table S1. After several sets of measurements (62 samples), we failed to detect levels of miR-488 and miR-920 in our plasma samples. We decided to remove these miRNAs from further analysis of the set of tested miRNAs. Similarly, we excluded miR-30a and miR-133a, which

Table 2 List of selected miRNAs

\begin{tabular}{|c|c|c|}
\hline Selected miRNA & Function related to hyperuricemia/gout & Reference \\
\hline miR-17-5 $p^{*}$ & suppresses NLRP3 inflammasome activation, miR-17-92 cluster & {$[17,18]$} \\
\hline miR-18a-5p* & increased by IL-1 $\beta$ in OA, miR-17-92 cluster & [19] \\
\hline miR-30a-3p & regulates the autoimmune responses occurring in RA & [20] \\
\hline miR-30c-5 $p^{*}$ & inhibits pyroptosis incurred by NLRP3 & [21] \\
\hline miR-126-3p & targets the CCL2 mRNA & [22] \\
\hline miR-133a-3p* & suppresses NLRP3 inflammasome activation & [23] \\
\hline miR-142-3p* & inhibits the expression of $A B C G 2$ & [24] \\
\hline miR-143-3p* & targets the GLUT9 mRNA & [25] \\
\hline miR-146a-5p & increased by MSU crystals, regulates the inflammatory response & [26] \\
\hline miR-155-5p & increased by MSU crystals, promotes the production of proinflammatory cytokines & [27] \\
\hline miR-222-3p* & targets the $A B C G 2$ mRNA & [28] \\
\hline miR-223-3p & reduces NLRP3 inflammasome activity & [14] \\
\hline miR-488-3p & regulates the production of proinflammatory cytokines, targets the ILIB mRNA, decreased at patients with GA & [29] \\
\hline miR-920 & regulates the production of proinflammatory cytokines, targets the IL1B mRNA, decreased at patients with GA & [29] \\
\hline
\end{tabular}

$O A$ osteoarthritis, RA rheumatoid arthritis, MSU monosodium urate, GA gouty arthritis

miRNAs signed with a * indicate significant differences in TLDA screening 


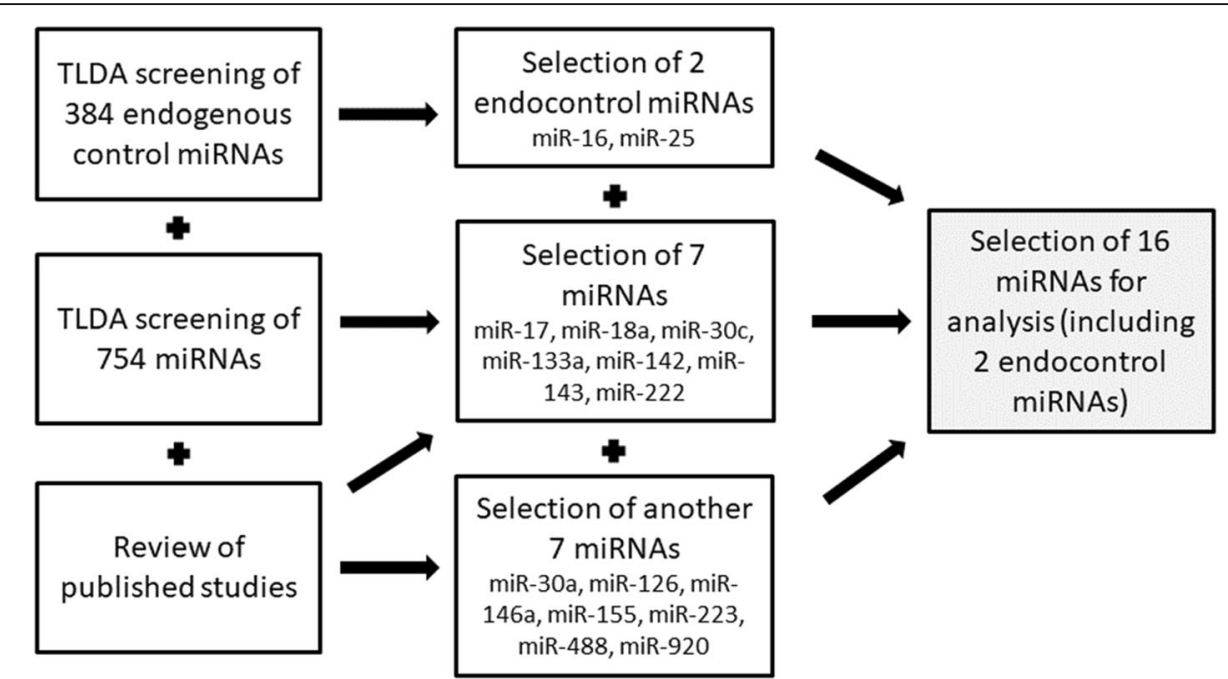

Fig. 1 Flowchart of miRNA selection. We chose two miRNAs as endogenous controls and 14 miRNAs for further analysis. The flowchart is based on a combination of TLDA card screening and a review of the literature

showed a high percentage ( $48 \%$ and $57 \%$, respectively) of undetectable or invalid data in our cohort.

Relative expression of all miRNAs was calculated using the delta cycle threshold (dCt) method. For normalization, we used endogenous control miRNA miR-25 that showed equal expression between samples since miR-16 could be influenced by hemolysis [31].

To compare $\mathrm{dCt}$ between normouricemic, hyperuricemic, gout, and gout attack groups, the non-parametric Kruskal-Wallis ANOVA test was used. $P$-values were adjusted for multiple comparisons using the BenjaminiHochberg method. Possible associations with the $A B C G 2$ p.Q141K genotype and various cytokine levels were explored using mixed linear regression models; $\mathrm{dCt}$ $\log 2$-transformed was used for a better fit and an individual random intercept factor was used to accommodate for the occasional repeated measurements. Post hoc pairwise comparisons of log2-transformed dCt values between study groups were performed using the Tukey method.

When using the Kruskal-Wallis ANOVA, we assumed independence between individual measurements. However, 46 individuals had two and one individual had four samples. To explore the influence of a possible dependence structure, we estimated the differences between cohorts using General Estimating Equations with both independence variance structure and unstructured variance settings, with $\mathrm{dCt} \log 2$-transformed as the response variable and the diagnostic group as an independent variable. Both variance models showed very little difference, justifying the use of the independence assumption.

All analyses were performed in statistical language and environment $\mathrm{R}$, version 4.0.2. The level of statistical significance was set to 0.05 .

\section{Results}

Comparison of miRNA levels between studied groups

We found five miRNAs (miR-17, miR-18a, miR-30c, miR-142, and miR-223) that showed significantly decreased expression in the normouricemic cohort compared to hyperuricemic, gout, and gout attack patients, in most cases.

Two miRNAs, belonging to the miR-17-92 cluster, miR-17 and miR-18a, were significantly more expressed in patients with hyperuricemia $(p<0.001$ and $p<0.028$, respectively), gout $(p<0.001)$, in for miR-17 also in gout attack patients $(p=0.002)$. These clustered miRNAs often showed a very similar trend in their expression.

The remaining miRNAs (miR-30c and miR-223) also had significantly higher expression levels for each of the studied groups compared to normouricemic controls ( $p$ $<0.001$ and $p<0.01$, respectively), and miR-142 showed the same trend as miR-18a $(p<0.01)$. A deviation of miR-18a and miR-142 in the gout attack cohort could be explained by the small number of patients in this group. Relevant $p$-values are graphically represented in Fig. 2. The remaining miRNAs (miR-30a, miR-126, miR-133a, miR-143, miR-146a, miR-155, miR-222, miR-488, and miR-920) did not vary in our analyzed groups. All results are listed in Supplementary Table S2.

\section{Association of miRNA levels and cytokines}

Our aim was to extend our previous study dealing with plasma cytokines in the same patients [16]. By using the same groups, we could take a closer look at the correlations between miRNA and cytokine levels. We found a correlation between low levels of MCP-1 and several miRNA levels. Levels of miR-17, miR-30c, miR-126, 


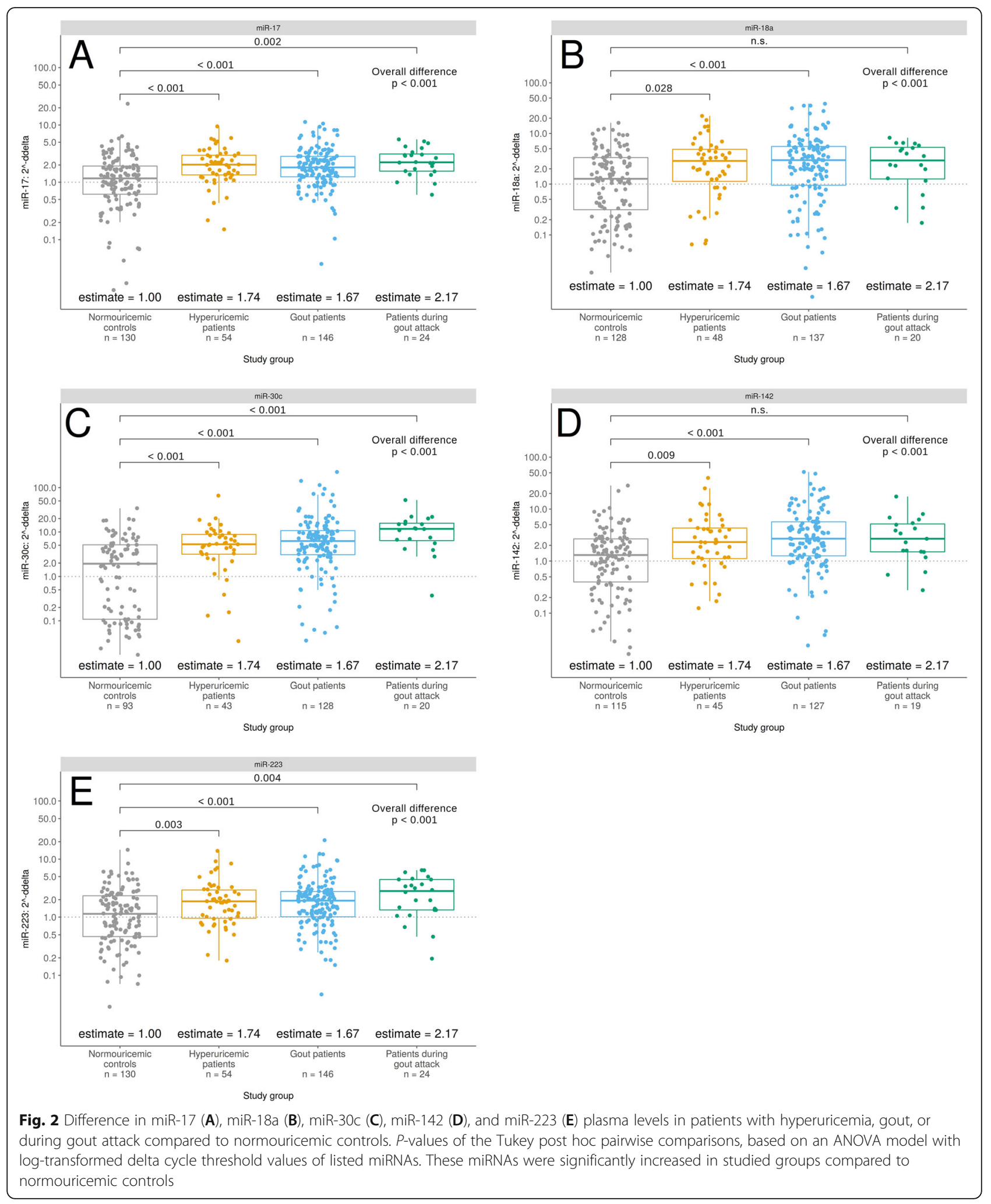


miR-142, and miR-223 were negatively correlated with levels of MCP-1. Results are plotted in Fig. 3.

\section{Correlation of miRNA levels and biochemical parameters}

Several biochemical parameters were measured in our patient groups (Table 1). We found positive correlations $(p<0.05)$ between CRP and all miRNA levels (except miR-143). Another positive correlation was between serum creatinine and levels of miR-126, miR-142, miR146a, miR-155, and miR-222 $(p<0.01)$; linked to these results, the same miRNAs were negatively correlated with eGFR. Other results are presented in Supplementary Table S3.

\section{Association of miRNA levels and $\mathrm{p} . \mathrm{Q} 141 \mathrm{~K}$ polymorphism in $A B C G 2$}

Based on the study that explained how the presence of the p.Q141K polymorphism changes the mRNA structure of the $A B C G 2$ gene and thus facilitates binding of miRNAs [32], we tried to examine if there was a difference in miRNA levels between carriers of the p.Q141K variant and wild-types. However, the association between miRNA levels and p.Q141K genotype was weak.

\section{Discussion}

Comparison of miRNA levels between studied groups

MiRNAs have been extensively studied in recent years, revealing evidence that miRNAs play a role in the pathophysiology of many diseases, including gout [33]. The first study related to miRNAs and gout was published seven years ago and worked on the assumption that miR-155 plays a key role in the proinflammatory activation of human myeloid cells and antigen-driven inflammatory arthritis [34]. They examined the role of miR155 in the acute phase of gout, which resulted in finding that miR-155 was upregulated in synovial fluid mononuclear cells in patients with acute gouty arthritis. This upregulation was negatively correlated with the expression of the SHIP-1 protein, which can increase the production of proinflammatory cytokines [27]. Thus the team of Yang et al. decided to verify these results in vivo using a mouse model; however, they did not find any significant differences in acute in vivo gout manifestation

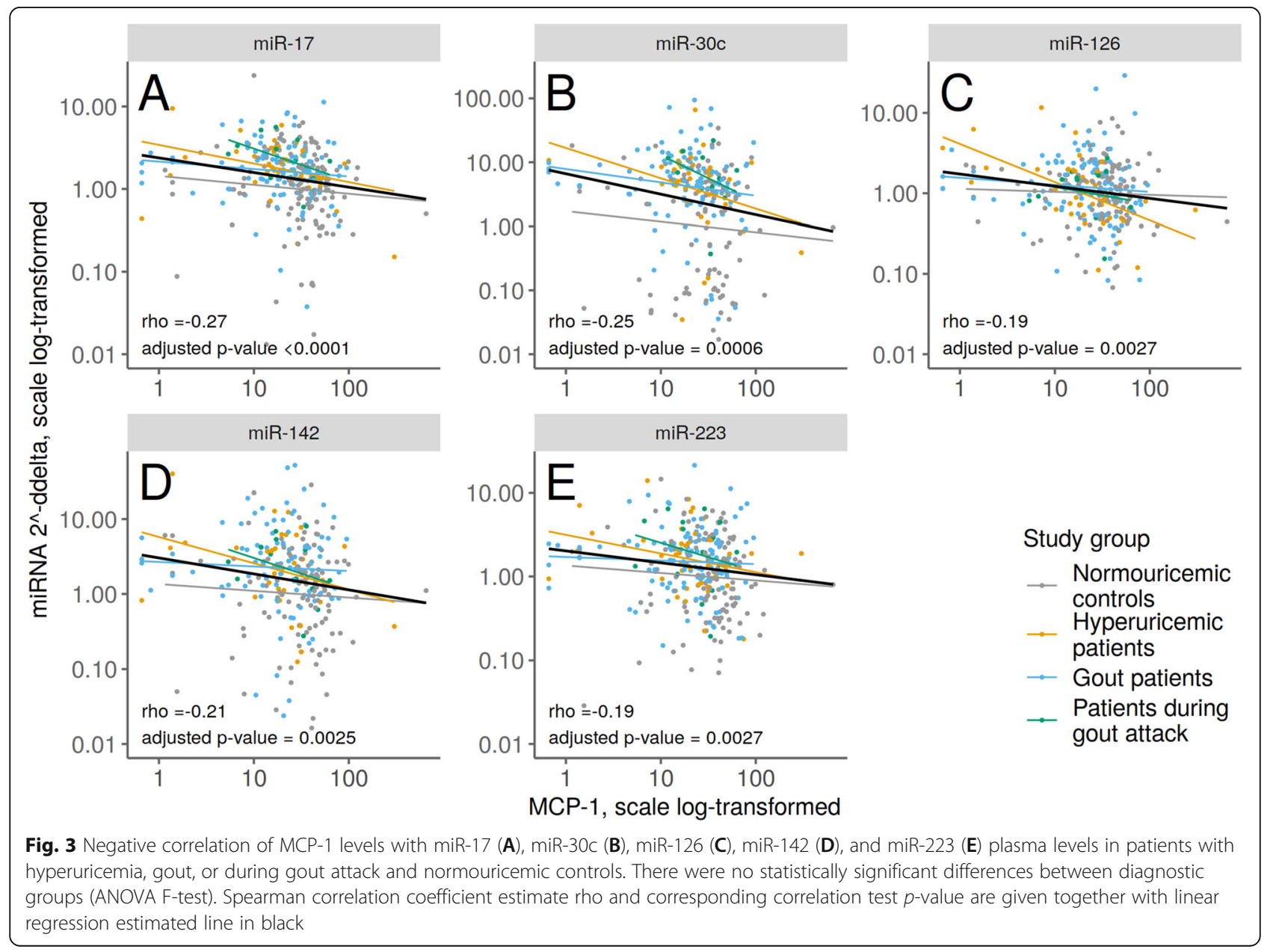


between diverse models [35]. In our study, we also did not find any significant differences in miR-155 plasma levels between the studied groups; there was only a slightly increasing trend with diagnosis progression. A study of the role of miR-146a in the acute inflammatory response to MSU crystals was created on the basis that miR-146a has been repeatedly described in connection with rheumatoid arthritis [36]. MSU crystals induce miR-146a expression in monocytic THP-1 cells, which results in inhibition of MSU crystal-induced proinflammatory cytokine gene expression in those cells. The peripheral blood mononuclear cells (PBMCs) of patients in the intercritical phase of gout expressed higher levels of miR-146a compared to the acute gout group and control group [26]. Our results did not show any differences within groups of patients with hyperuricemia, gout, or a gout attack, not even compared to normouricemic controls.

Because the proinflammatory cytokine IL- $1 \beta$ is crucial for inflammation, as well as in acute gouty arthritis, there have been several studies addressing its regulation by miRNAs. Zhou et al. discovered five miRNAs (miR30c-1-3p, miR-488-3p, miR-550a-3p, miR-663a, and miR-920) that possibly target IL-1 $\beta$; furthermore, they demonstrated significantly decreased levels of miR-488 and miR-920 in the peripheral white blood cells of patients with acute gouty arthritis. They showed that levels of IL- $1 \beta$ were significantly higher in these patients. They also described the effects of MSU crystals on the inhibited expression of miR-488 and miR-920 and induced mRNA expression of proinflammatory cytokines, such as IL- $1 \beta$, IL- 8 , and TNF- $\alpha$ in monocytic THP-1 cells. In addition, they demonstrated direct targeting of IL-1 $\beta$ 3' UTR by miR-488 and miR-920 [29]. After several sets of measurements (62 samples), we failed to detect altered levels of these miRNAs in our plasma samples. These miRNAs are probably not released into the extracellular environment. During further analysis, we decided to remove these miRNAs from the set of tested miRNAs. In a similar way, we excluded miR-30a and miR-133a since both showed a high percentage ( $48 \%$ and $57 \%$, respectively) of undetectable or invalid data in our groups of patients. We discontinued the analysis of these miRNAs in the course of our research.

The expression of miR-18a is induced by IL- $1 \beta$ and accelerates the progression of osteoarthritis [19]. IL-1 $\beta$ is a pivotal cytokine in the inflammatory gout cascade; our results are consistent when we consider differences between groups where patients with hyperuricemia and gout had significantly higher levels of miR-18a; however, we did not find any difference for gout attack patients or a direct correlation between IL-1 $\beta$ and miR-18a levels.

The miR-17-92 cluster, including miR-17 and miR18a, plays a role in oncogenesis [37] and proliferation and activation of B-cells, T-cells, and macrophages [38]. We found a significantly higher expression of miR-17 and miR-18a in groups of patients with hyperuricemia and gout, and in the case of miR-17, we found higher expression in patients experiencing a gout flare in comparison with the normouricemic group. miR-17-5p can also deactivate NLRP3 inflammasomes through binding and decreasing the thioredoxin-interacting protein (TXNIP) mRNA $[17,18]$.

There many studies on NLRP3 inflammasome and its regulation by miRNAs; it is a key component not only in gout but also in other inflammatory diseases. In a recent summary review by Zamani et al., they present a list of 20 miRNAs associated with NLRP3 regulation, including miR-17-5p, miR-133a-1, miR-146a, miR-155, and miR223 [39]. miR-223 was described earlier as a myeloidspecific miRNA capable of suppressing NLRP3 expression, which leads to reduced NLRP3 inflammasome activity [14]. Our data showed upregulation of miR-223 in the hyperuricemia, gout, and gout flare patients. miR223 targets and negatively regulates NLRP3 expression and controls inflammasome activation in macrophages [14]. Upregulation of miR-223 in our plasma samples of patients with hyperuricemia, gout, and gout flare could indicate persistent inflammation even during hyperuricemia.

Experiments using human aortic endothelial cells (HAEC) revealed that miR-30c-5p could inhibit pyroptosis incurred by NLRP3 via FOXO3 targeting [21]. FOXO3 is a transcription factor associate with serum uric acid levels [40]. Our data show upregulation of this miRNA in patients with hyperuricemia, gout, and during a gout attack.

We also tried to analyze miR-302f in one set of measurements, but all samples in the set showed undetectable levels of this miRNA. Our results did not help clarify the role of miR-302f as a potential gout locus [7].

\section{Correlation of miRNA levels and biochemical parameters}

We found correlations between several miRNAs and clinical/biochemical parameters such as BMI, serum uric acid, fractional uric acid excretion, glomerular filtration rate, serum creatinine, and CRP. Almost all miRNA levels were positively correlated with CRP levels. Higher levels of CRP are associated with the acute-phase of inflammation. In our previous study, we showed higher CRP levels in patients with hyperuricemia and gout; however, those patients who were not carriers of the p.Q141K variant of the $A B C G 2$ gene had significantly lower CRP levels [16]. This could be a possible explanation for the results of an earlier study where CRP levels were not connected with hyperuricemia [41]. There are few studies describing an association between circulating miRNA and CRP levels; for example, a positive 
correlation between miR-155 and CRP was recently published [42]. Five miRNAs (miR-126, miR-142, miR-146a, miR-155, and miR-222) showed a positive correlation with serum creatinine and, therefore, a negative correlation with eGFR. Higher levels of serum creatinine and, therefore, lower values of eGFR are associated with abnormal renal function, which is also a risk factor for gout [43].

\section{Association of miRNA levels and cytokines}

We were interested in the association between levels of miRNA and cytokines. In our previous study, we examined the association between 27 cytokines with disease level. We found negative correlations between MCP-1 levels and miR-17, miR-30c, miR-126, miR-142, and miR-223 levels. Chemokine MCP-1 (monocyte chemoattractant protein-1) is a chemotactic factor for monocytes. Uric acid increases MCP-1 production, which is an essential part of the immune response to hyperuricemia and gout [44, 45]. We did not confirm this in our cohort of hyperuricemia and gout patients; in fact, the normouricemic control group showed significantly higher levels of MCP-1 in plasma samples [16]. On the other hand, we showed a negative correlation between this cytokine and several miRNAs that could possibly influence MCP-1 expression. According to the online miRNA target database (http://www.mirdb.org/), there are 46 target miRNAs for the CCL2 gene (equal to MCP-1), but none of them are coincident with our miRNA selection. However, miR-126 can bind to 3'UTR of CCL2, according to the study investigating CCL2 production in white adipose tissue inflammation. Furthermore, this study did not reveal any effect of miR-30c on adipocyte CCL2 secretion [22]. Also, miR-223 overexpression decreased several cytokines, including MCP-1 in glioblastoma cell lines [46]. On the contrary, overexpression of miR-142 increased CCL2 levels in monocyte-derived dendritic cells [47]. Other of the published miRNAs associated with MCP-1 are for example miR-124a [48], miR-122 [49], miR-421 [50], and miR374a [51]. More studies are necessary in order to clarify all possible miRNAs targeting MCP-1.

\section{Association of miRNA levels and $\mathrm{p} . \mathrm{Q} 141 \mathrm{~K}$ polymorphism in $A B C G 2$}

One of the first studies in the field of oncology showed that RNA interference with the $A B C G 2$ gene could downregulate gene expression and modulate the functional phenotype of cells [52]. Importantly, a study connecting our previous and current research describes the impact of the p.Q141K polymorphism on miRNAdependent $A B C G 2$ repression. The study concluded that the presence of the $\mathrm{p} . \mathrm{Q} 141 \mathrm{~K}$ polymorphism alters the secondary structure of $A B C G 2 \mathrm{mRNA}$ and facilitates translational repression by miRNAs [32]. However, we did not find any significant association between the studied miRNAs and the p.Q141K polymorphism in our groups. MiR-142-3p binds to 3'UTR and the coding sequence of $A B C G 2$ and inhibits its expression [24]. We reported upregulation of miR-142-3p in the plasma of hyperuricemic, gout, and gout flare patients. Moreover, a recent study reported that miR-143-3p could directly target GLUT9 (SLC2A9 gene). MiR-143-3p was significantly reduced in kidney tissues from a hyperuricemia mice model. They also confirmed this result in humans, where miR-143-3p was significantly more expressed in the serum of healthy controls compared to hyperuricemic patients [25]. We did not find any significant differences in miR-143-3p levels between the studied groups.

\section{Conclusions}

In conclusion, plasma levels of several analyzed miRNAs (miR-17, miR-18a, miR-30c, miR-142, and miR-223) were upregulated in hyperuricemic, gout, and gout attack patients compared to normouricemic controls. Unfortunately, we did not find any differences in miRNA levels between particular stages of the disease, i.e., hyperuricemia, gout, and gout attack. On the other hand, we found negative correlations between several miRNAs (miR-17, miR-30c, miR-126, miR-142, and miR-223) and plasma chemokine MCP-1 levels. Furthermore, a positive correlation between CRP and all analyzed miRNAs (except miR-143) was noticed. Five of those miRNAs (miR-126, miR-142, miR-146a, miR-155, and miR-222) also showed a positive correlation with serum creatinine and, therefore, a negative correlation with eGFR.

\section{Abbreviations}

ABCG2: ATP-binding cassette super-family G member 2; BMl: Body mass index; CCL2: C-C Motif Chemokine Ligand 2; Cq: Quantification cycle; CRP: Creactive protein; dCt: Delta cycle threshold; eGFR: Estimated glomerular filtration rate; FOXO3: Forkhead Box O3; GLUT9: Glucose Transporter Type 9; GWAS: Genome-wide association studies; HAEC: Human aortic endothelial cells; IL-1 $\beta$ : Interleukin-1 $\beta$; IL-6: Interleukin-6; IL-8: Interleukin-8; IQR: Interquartile range; MCP-1: Monocyte chemoattractant protein-1; MICE: Multivariate Imputation by Chained Equations; MSU: Monosodium urate; NLRP3: NLR Family Pyrin Domain Containing 3; OMIM: Online Mendelian Inheritance in Man; PBMCs: Peripheral blood mononuclear cells; qPCR: Quantitative PCR; SLC22A12: Solute Carrier Family 22 Member 12; SLC2A9: Solute Carrier Family 2 Member 9; SUA: Serum uric acid; THP-1 cells: Human acute monocytic leukemia cell line; TLDA: TaqMan Low Density Arrays; TNF-a: Tumor necrosis factor a; TXNIP: Thioredoxin-interacting protein; URAT1: Urate Transporter 1; UTR: Untranslated region

\section{Supplementary Information}

The online version contains supplementary material available at https://doi. org/10.1186/s13075-021-02569-w.

Additional file 1: Supplementary Table S1. List of all undetectable, valid, and invalid measurements. Supplementary Table S2. Comparison of delta cycle threshold (dCt) miRNA values between studied groups. Supplementary Table S3. $P$-values of miRNA levels and biochemical parameters correlations. 


\section{Acknowledgements}

We are grateful to all the patients who took part in this study as well as our colleagues at the Institute of Rheumatology for their help in recruiting patients for the study (namely Jakub Závada, Karel Pavelka, Lenka Hasíková, and Pavel Ješina).

\section{Authors' contributions}

Study conception and design: BS and JB; acquisition of data: JB and VH; analysis and interpretation of data: BS, JB, and MP. All authors were involved in drafting the manuscript or revising it critically for content. All the authors approved the final version for publication.

\section{Funding}

This work was supported by a grant from the Czech Republic Ministry of Health RVO 00023728 (Institute of Rheumatology), RVO VFN64165, BBMRI-CZ LM2018125, and by Charles University SW 260367 UK.

\section{Availability of data and materials}

The datasets used and/or analyzed during the current study are available from the corresponding author on reasonable request.

\section{Declarations}

\section{Consent for publication}

Not applicable.

\section{Competing interests}

The authors declare that they have no competing interests.

\begin{abstract}
Author details
${ }^{1}$ Institute of Rheumatology, Prague, Czech Republic. ${ }^{2}$ Department of Rheumatology, First Faculty of Medicine, Charles University, Prague, Czech Republic. ${ }^{3}$ Faculty of Science, Charles University, Prague, Czech Republic. ${ }^{4}$ Department of Probability and Mathematical Statistics, Faculty of Mathematics and Physics, Charles University, Prague, Czech Republic. ${ }^{5}$ Department of Pediatrics and Inherited Metabolic Disorders, First Faculty of Medicine, Charles University and General University Hospital, Prague, Czech Republic.
\end{abstract}

\section{Received: 22 March 2021 Accepted: 28 June 2021}

Published online: 10 July 2021

\section{References}

1. Hurba O, Mancikova A, Krylov V, Pavlikova M, Pavelka K, Stibůrková B. Complex analysis of urate Transporters SLC2A9, SLC22A12 and functional characterization of non-synonymous allelic variants of GLUT9 in the Czech population: no evidence of effect on hyperuricemia and gout. PLoS One. 2014;9. https://doi.org/10.1371/journal.pone.0107902

2. Matsuo H, Ichida K, Takada T, Nakayama A, Nakashima H, Nakamura T, et al. Common dysfunctional variants in ABCG2 are a major cause of early-onset gout. Sci Rep. 2013;3:8-11. https://doi.org/10.1038/srep02014.

3. Stiburkova B, Pavelcova K, Zavada J, Petru L, Simek P, Cepek P, et al. Functional non-synonymous variants of $A B C G 2$ and gout risk. Rheumatol (United Kingdom). 2017;56:1982-92. https://doi.org/10.1093/rheumatology/ kex295.

4. Toyoda Y, Pavelcová K, Klein M, Suzuki H, Takada T, Stiburkova B. Familial early-onset hyperuricemia and gout associated with a newly identified dysfunctional variant in urate transporter ABCG2. Arthritis Res Ther. 2019;21: 219. https://doi.org/10.1186/s13075-019-2007-7.

5. Perez-Ruiz F, Marimon E, Chinchilla SP. Hyperuricaemia with deposition: latest evidence and therapeutic approach. Ther Adv Musculoskelet Dis. 2015;7:225-33. https://doi.org/10.1177/1759720X15599734.

6. Dalbeth N, Choi HK, Joosten LAB, Khanna PP, Matsuo H, Perez-Ruiz F, et al. Gout. Nat Rev Dis Prim. 2019;5:69. https://doi.org/10.1038/s41572019-0115-y.

7. Kawamura Y, Nakaoka H, Nakayama A, Okada Y, Yamamoto K, Higashino T, et al. Genome-wide association study revealed novel loci which aggravate asymptomatic hyperuricaemia into gout. Ann Rheum Dis. 2019;78:1430-7. https://doi.org/10.1136/annrheumdis-2019-215521.
8. Lee RC, Feinbaum RL, Ambros V. The C. elegans heterochronic gene lin-4 encodes small RNAs with antisense complementarity to lin-14. Cell. 1993;75: 843-54. https://doi.org/10.1016/0092-8674(93)90529-y.

9. Sohel MH. Extracellular/Circulating MicroRNAs: Release Mechanisms, Functions and Challenges. Achiev Life Sci. Elsevier BV. 2016;10:175-86. https://doi.org/10.3390/ijms22020542.

10. Mi S, Zhang J, Zhang W, Huang RS. Circulating MicroRNAs as Biomarkers for Inflammatory Diseases. MicroRNA. 2013;2:64-72. https://doi.org/10.21 74/2211536611302010007.

11. Friedman RC, Farh $\mathrm{KKH}$, Burge CB, Bartel DP. Most mammalian mRNAs are conserved targets of microRNAs. Genome Res. Cold Spring Harbor Laboratory Press. 2009;19:92-105. https://doi.org/10.1101/gr.082701.108.

12. Sun WF, Zhu MM, Li J, Zhang XX, Liu YW, Wu XR, et al. Effects of Xie-ZhuoChu-Bi-Fang on miR-34a and URAT1 and their relationship in hyperuricemic mice. J Ethnopharmacol. Elsevier Ireland Ltd. 2015;161:163-9. https://doi. org/10.1016/j.jep.2014.12.001.

13. Knake C, Stamp L, Bahn A. Molecular mechanism of an adverse drug-drug interaction of allopurinol and furosemide in gout treatment. Biochem Biophys Res Commun. Elsevier Inc. 2014;452:157-62. https://doi.org/10.1016/ j.bbrc.2014.08.068

14. Bauernfeind F, Rieger A, Schildberg FA, Knolle PA, Schmid-Burgk JL, Hornung V NLRP3 inflammasome activity is negatively controlled by miR-223. J Immunol. 2012;189:4175-81. https://doi.org/10.4049/jimmunol.1201516.

15. Wallace SL, Robinson H, Masi AT, Decker JL, Mccarty DJ, Yü TF. Preliminary criteria for the classification of the acute arthritis of primary gout. Arthritis Rheum. 1977;20:895-900. https://doi.org/10.1002/art.1780200320.

16. Horváthová V, Bohatá J, Pavlíková M, Pavelcová K, Pavelka K, Šenolt L, et al. Interaction of the p.Q141K variant of the ABCG2 gene with clinical data and cytokine levels in primary hyperuricemia and gout. J Clin Med. 2019:8:1965. https://doi.org/10.3390/jcm8111965.

17. Lerner AG, Upton JP, Praveen PVK, Ghosh R, Nakagawa Y, Igbaria A, et al. IRE1a induces thioredoxin-interacting protein to activate the NLRP3 inflammasome and promote programmed cell death under irremediable ER stress. Cell Metab. 2012;16:250-64. https://doi.org/10.1016/j.cmet.2012.07.007.

18. Chen D, Dixon BJ, Doycheva DM, Li B, Zhang Y, Hu Q, et al. IRE1a inhibition decreased TXNIP/NLRP3 inflammasome activation through miR-17-5p after neonatal hypoxic-ischemic brain injury in rats. J Neuroinflammation. 2018; 15:32. https://doi.org/10.1186/s12974-018-1077-9.

19. Lian C, Tao T, Su P, Liao Z, Wang X, Lei Y, et al. Targeting miR-18a sensitizes chondrocytes to anticytokine therapy to prevent osteoarthritis progression. Cell Death Dis. 2020;11:947. https://doi.org/10.1038/s41419-020-03155-9.

20. Alsaleh G, François A, Philippe L, Gong YZ, Bahram S, Cetin S, et al. MiR-30a$3 p$ negatively regulates BAFF synthesis in systemic sclerosis and rheumatoid arthritis fibroblasts. PLoS One. Public Library of Science. 2014;9. https://doi. org/10.1371/journal.pone.0111266.

21. Li P, Zhong $X$, Li J, Liu H, Ma X, He R, et al. MicroRNA-30c-5p inhibits NLRP3 inflammasome-mediated endothelial cell pyroptosis through $\mathrm{FOXO} 3$ downregulation in atherosclerosis. Biochem Biophys Res Commun. 2018;503: 2833-40. https://doi.org/10.1016/j.bbrc.2018.08.049.

22. Arner E, Mejhert N, Kulyté A, Balwierz PJ, Pachkov M, Cormont M, et al. Adipose tissue MicroRNAs as regulators of $C C L 2$ production in human obesity. Diabetes American Diabetes Association. 2012;61:1986-93. https:// doi.org/10.2337/db11-1508.

23. Bandyopadhyay S, Lane T, Venugopal R, Parthasarathy PT, Cho Y, Galam L, et al. MicroRNA-133a-1 regulates inflammasome activation through uncoupling protein-2. Biochem Biophys Res Commun. NIH Public Access. 2013;439:407-12. https://doi.org/10.1016/j.bbrc.2013.08.056.

24. Shen WW, Zeng Z, Zhu WX, Fu GH. MiR-142-3p functions as a tumor suppressor by targeting CD133, ABCG2, and Lgr5 in colon cancer cells. J Mol Med. 2013;91:989-1000. https://doi.org/10.1007/s00109-013-1037-X.

25. Zhou Z, Dong Y, Zhou H, Liu J, Zhao W. MiR-143-3p directly targets GLUT9 to reduce uric acid reabsorption and inflammatory response of renal tubular epithelial cells. Biochem Biophys Res Commun. 2019;517:413-20. https://doi. org/10.1016/j.bbrc.2019.07.114.

26. Dalbeth N, Pool B, Shaw OM, Harper JL, Tan P, Franklin C, et al. Role of miR$146 a$ in regulation of the acute inflammatory response to monosodium urate crystals. Ann Rheum Dis. 2015;74:786-90. https://doi.org/10.1136/a nnrheumdis-2014-205409.

27. Jin HM, Kim TJ, Choi JH, Kim MJ, Cho YN, Nam K. II, et al. MicroRNA-155 as a proinflammatory regulator via SHIP-1 down-regulation in acute gouty arthritis. Arthritis Res Ther. 2014;16:R88. https://doi.org/10.1186/ar4531. 
28. Zhao L, Ren Y, Tang H, Wang W, He Q, Sun J, et al. Deregulation of the miR222-ABCG2 regulatory module in tongue squamous cell carcinoma contributes to chemoresistance and enhanced migratory/invasive potential. Oncotarget. Impact Journals LLC. 2015;6:44538-50. https://doi.org/10.18632/ oncotarget.6253.

29. Zhou W, Wang Y, Wu R, He Y, Su Q, Shi G. MicroRNA-488 and -920 regulate the production of proinflammatory cytokines in acute gouty arthritis. Arthritis Res Ther. 2017:19:203. https://doi.org/10.1186/s13075-017-1418-6.

30. de Ronde MWJ, Ruijter JM, Lanfear D, Bayes-Genis A, Kok MGM, Creemers $\mathrm{EE}$, et al. Practical data handling pipeline improves performance of qPCRbased circulating miRNA measurements. RNA. 2017;23:811-21. https://doi. org/10.1261/rna.059063.116.

31. Kirschner MB, Edelman JJB, Kao SCH, Vallely MP, Van Zandwijk N, Reid G. The impact of hemolysis on cell-free microRNA biomarkers. Front Genet. 2013:4:94. https://doi.org/10.3389/fgene.2013.00094.

32. Ripperger A, Benndorf RA. The C421A (Q141K) polymorphism enhances the 3'-untranslated region (3'-UTR)-dependent regulation of ATP-binding cassette transporter ABCG2. Biochem Pharmacol. 2016;104:139-47. https:// doi.org/10.1016/j.bcp.2016.02.011.

33. Wang $Y, X u D$, Wang $B$, Hou $X$. Could microRNAs be regulators of gout pathogenesis? Cell Physiol Biochem. 2015;36:2085-92. https://doi.org/10.11 59/000430176.

34. Kurowska-Stolarska M, Alivernini S, Ballantine LE, Asquith DL, Millar NL, Gilchrist DS, et al. MicroRNA-155 as a proinflammatory regulator in clinical and experimental arthritis. Proc Natl Acad Sci U S A. 2011;108:11193-8. https://doi.org/10.1073/pnas.1019536108.

35. Yang Q, Zhang Q, Qing Y, Zhou L, Mi Q, Zhou J. miR-155 is dispensable in monosodium urate-induced gouty inflammation in mice. Arthritis Res Ther. 2018;20:177. https://doi.org/10.1186/s13075-018-1550-y.

36. Bae SC, Lee YH. MiR-146a levels in rheumatoid arthritis and their correlation with disease activity: a meta-analysis. Int J Rheum Dis. 2018;21:1335-42. https://doi.org/10.1111/1756-185X.13338.

37. He L, Thomson JM, Hemann MT, Hernando-Monge E, Mu D, Goodson S, et al. A microRNA polycistron as a potential human oncogene. Nature. 2005; 435:828-33. https://doi.org/10.1038/nature03552.

38. Kuo G, Wu CY, Yang HY. MiR-17-92 cluster and immunity. J Formos Med Assoc. 2019;118:2-6. https://doi.org/10.1016/j.jfma.2018.04.013.

39. Zamani P, Oskuee RK, Atkin SL, Navashenaq JG, Sahebkar A. MicroRNAs as important regulators of the NLRP3 inflammasome. Prog Biophys Mol Biol. 2020;150:50-61. https://doi.org/10.1016/j.pbiomolbio.2019.05.004.

40. Lang S, Hilsabeck TA, Wilson KA, Sharma A, Bose N, Brackman DJ, et al. A conserved role of the insulin-like signaling pathway in diet-dependent uric acidpathologies in Drosophila melanogaster. PLoS Genet. 2019;15:e1008318. https://doi.org/10.1371/journal.pgen.1008318.

41. Okuda C, Koyama H, Tsutsumi Z, Yamamoto A, Kurajoh M, Moriwaki Y, et al. Serum CRP in patients with gout and effects of benzbromarone. Int J Clin Pharmacol Ther. Int J Clin Pharmacol Ther. 2011;49:191-7. https://doi.org/10. 5414/cp201425.

42. Su Q, Yang H, Li L. Circulating miRNA-155 as a potential biomarker for coronary slow flow. Dis Markers. 2018;2018:6345284. https://doi.org/10.11 55/2018/6345284.

43. Krishnan E. Reduced Glomerular Function and Prevalence of Gout: NHANES 2009-10. PLoS One. 2012;7:e50046. https://doi.org/10.1371/journal.pone. 0050046.

44. Kanellis J, Watanabe S, Li JH, Kang DH, Li P, Nakagawa T, et al. Uric acid stimulates monocyte chemoattractant protein-1 production in vascular smooth muscle cells via mitogen-activated protein kinase and cyclooxygenase-2. Hypertension. 2003;41:1287-93. https://doi.org/10.1161/ 01.HYP.0000072820.07472.3B

45. Grainger R, McLaughlin RJ, Harrison AA, Harper JL. Hyperuricaemia elevates circulating CCL2 levels and primes monocyte trafficking in subjects with inter-critical gout. Rheumatol (Oxford). 2013;52:1018-21. https://doi.org/10.1 093/rheumatology/kes326.

46. Ding Q, Shen L, Nie X, Lu B, Pan X, Su Z, et al. MiR-223-3p overexpression inhibits cell proliferation and migration by regulating inflammationassociated cytokines in glioblastomas. Pathol Res Pract. Elsevier. 2018;214: 1330-9. https://doi.org/10.1016/j.prp.2018.05.012.

47. Wang Y, Liang J, Qin H, Ge Y, Du J, Lin J, et al. Elevated expression of miR$142-3 p$ is related to the pro-inflammatory function of monocyte-derived dendritic cells in SLE. Arthritis Res Ther. BioMed Central Ltd. 2016:18:1-11. https://doi.org/10.1186/s13075-016-1158-z.
48. Nakamachi Y, Kawano S, Takenokuchi M, Nishimura K, Sakai Y, Chin T, et al. MicroRNA-124a is a key regulator of proliferation and monocyte chemoattractant protein 1 secretion in fibroblast-like synoviocytes from patients with rheumatoid arthritis. Arthritis Rheum. 2009;60:1294-304. https://doi.org/10.1002/art.24475.

49. Liao M-F, Hsu J-L, Lu K-T, Chao P-K, Cheng M-Y, Hsu H-C, et al. Granulocyte Colony Stimulating Factor (GCSF) Can Attenuate Neuropathic Pain by Suppressing Monocyte Chemoattractant Protein-1 (MCP-1) Expression, through Upregulating the Early MicroRNA-122 Expression in the Dorsal Root Ganglia. Cells. 2020;9:1669. https://doi.org/10.3390/cells9071669.

50. Zhu F, Yin J, Li J, Xue J. MicroRNA-421 affects the chemotaxis of monocytes via MCP-1, and regulates the local immune responses in injured cartilage site of elbow joint of upper limbs. Biotechnol Biotechnol Equip. 2020;34: 294-302. https://doi.org/10.1080/13102818.2020.1738955.

51. Yang Z, Guo Z, Dong J, Sheng S, Wang Y, Yu L, et al. miR-374a regulates inflammatory response in diabetic nephropathy by targeting MCP-1 expression. Front Pharmacol. 2018;9:900. https://doi.org/10.3389/fphar.2018. 00900.

52. Ee PLR, He X, Ross DD, Beck WT. Modulation of breast cancer resistance protein (BCRP/ABCG2) gene expression using RNA interference. Mol Cancer Ther. 2004;3:1577-83 PMID: 15634651

\section{Publisher's Note}

Springer Nature remains neutral with regard to jurisdictional claims in published maps and institutional affiliations.
Ready to submit your research? Choose BMC and benefit from:

- fast, convenient online submission

- thorough peer review by experienced researchers in your field

- rapid publication on acceptance

- support for research data, including large and complex data types

- gold Open Access which fosters wider collaboration and increased citations

- maximum visibility for your research: over $100 \mathrm{M}$ website views per year

At BMC, research is always in progress.

Learn more biomedcentral.com/submissions 